University of New Hampshire

University of New Hampshire Scholars' Repository

Physics Scholarship

Physics

8-1-1987

\title{
Static magnetic field models consistent with nearly isotropic plasma pressure
}

Harlan E. Spence

Boston University, harlan.spence@unh.edu

Margaret G. Kivelson

Raymond J. Walker

Follow this and additional works at: https://scholars.unh.edu/physics_facpub

Part of the Physics Commons

\section{Recommended Citation}

Spence, Harlan E.; Kivelson, Margaret G.; and Walker, Raymond J., "Static magnetic field models consistent with nearly isotropic plasma pressure" (1987). Geophysical Research Letters. 300.

https://scholars.unh.edu/physics_facpub/300

This Article is brought to you for free and open access by the Physics at University of New Hampshire Scholars' Repository. It has been accepted for inclusion in Physics Scholarship by an authorized administrator of University of New Hampshire Scholars' Repository. For more information, please contact Scholarly.Communication@unh.edu. 


\title{
STATIC MAGNETIC FIELD MODELS CONSISTENT WITH NEARLY ISOTROPIC PLASMA PRESSURE
}

\author{
Harlan E. Spence ${ }^{1}$, Margaret G. Kivelson ${ }^{1,2}$, Raymond J. Walker ${ }^{2}$
}

Abstract. Using the empirical magnetospheric magnetic field models of Tsyganenko and Usmanov (TU), we have determined the self-consistent plasma pressure gradients and anisotropies along the midnight meridian in the near-Earth magnetosphere. By "inverting" the magnetic field, we determine what distributions of an anisotropic plasma, confined within the specified magnetic field configuration, are consistent with the magnetohydrostatic equilibrium condition, $\mathbf{J} \times \mathbf{B}=\nabla \cdot \mathbf{P}$. The TU model, parameterized for different levels of geomagnetic activity by the $K_{p}$ index, provided the magnetic field values from which $\mathbf{J} \times \mathbf{B}$ was numerically evaluated. $A$ best fit solution was found that minimized the average difference between $\mathbf{J} \times \mathbf{B}$ and $\nabla \cdot \mathbf{P}$ along an entire flux tube. Unlike previous semi-empirical models, the TU models contain magnetic stresses that can be balanced by a nearly isotropic plasma pressure with a reasonable radial gradient at the equator.

\section{Introduction}

A realistic magnetic field model must satisfy a number of criteria for it to be valid and/or useful. A practical consideration is that it must be easy to implement, but still be flexible enough to reflect seasonal, diurnal, and local time variations. The residual error between the observed vector magnetic field values and the model values should be small. Also, the model field must be divergenceless. A more subtle constraint is that the magnetic stresses of the model must be self-consistent with the distribution of mechanical forces in the magnetosphere. Specifically, during magnetically quiet intervals when the magnetohydrostatic equilibrium condition is approached, the magnetic stresses should be balanced predominantly by the plasma pressure gradients. An ideal magnetic field model would contain magnetospheric currents consistent with in situ data from both plasma and field experiments, rather than explicitly specified tail, ring, and magnetopause currents fit by field data alone [Voigt, 1981]. In virtually all empirical models to date, this constraint has not been imposed. Walker and Southwood [1982] showed that in many models the Maxwell stresses cannot balance an isotropic plasma pressure. Because of the failure of these models to be in pressure balance with an isotropic plasma, some contend (c.f. Voigt [1986]) that such semiempirical models are theoretically unsatisfactory.

The Tsyganenko and Usmanov models [1982] are new

\footnotetext{
${ }^{1}$ Department of Earth and Space Sciences, University of California, Los Angeles

2 Institute of Geophysics and Planetary Physics, University of California, Los Angeles
}

Copyright 1987 by the American Geophysical Union.

Paper number 7L6555.

0094-8276/87/007L-6555\$03.00 empirical magnetic field models whose consistency with pressure balance has not been tested. If a model represents the static global magnetospheric currents with some accuracy, one expects to be able to determine a (possibly) non-isotropic plasma distribution that is consistent with the magnetic field. In this paper, we present a technique for such a magnetic field "inversion" and use it to test the recent empirical magnetic field models. We will show that these models are consistent, in well-constrained regions, with a nearly isotropic plasma pressure in magnetohydrostatic equilibrium with magnetic stresses.

\section{Description of the Field Models}

The models used in this study were developed by Tsyganenko and Usmanov [1982]; we refer to them as the TU models. A brief summary of the TU models follows. The reader is urged to consult Tsyganenko and Usmanov [1982] for a more detailed discussion.

$\mathrm{TU}$ chose analytic formula to represent the separate magnetic field contributions of the internal field, the ring current, the cross-magnetotail current, and the magnetopause current. The internal field is represented by the harmonic expansion of the earth's internal field. The model ring current is an axially symmetric torus of westward directed current oriented with its normal parallel to the dipole magnetic moment. The cross-magnetotail current flows in a sheet across the tail; the current sheet has a finite thickness, varies in shape and magnitude along the tail axis, and merges with the ring current in the nearmagnetotail region. An empirical neutral sheet model is used to account for dipole tilt effects.

Unlike the ring current and magnetotail current systems, the magnetopause shape is not easily determined and the current distribution is complex. TU chose to represent the field of the magnetopause currents by nonlinear power series. Since the representation is not curlfree interior to the boundary, it accounts not only for the magnetopause currents, but also for any errors introduced by the ring and tail current representations as well as any remaining magnetospheric currents. The superposition of the three aformentioned external current systems and the internal field yields the full model magnetic field. Relationships among the model parameters guarantee a divergenceless magnetic field.

The empirical magnetic field models were fit to nearly 19,000 in situ vector field averages from the merged IMP and HEOS data sets. TU separated the data into 11 subsets according to the $K_{p}$ index. TU fit the model parameters to the data sets by an iterative minimization technique, creating models representative of geomagnetic conditions ranging from very quiet $\left(K_{p}=0\right)$ to disturbed $\left(K_{p}>3^{+}\right)$. The TU models should not be used in regions where few data were available to constrain the fitting of the model parameters. Owing to magnetometer saturation and orbital bias of the IMP and HEOS spacecraft, 
relatively little data were available inside of about $6 R_{E}$ and at magnetic latitudes greater than about $76^{\circ}$, or outside of about $20 R_{E}$ at distances near to or beyond the spacecraft apogees. We have restricted our analysis to areas that are within well-constrained regions.

\section{Momentum Balance in the Magnetohydrostatic Limit}

The large scale structure of the magnetosphere can be described by using Maxwell's equations and the magnetohydrodynamic (MHD) equations, so we use these equations to determine the self-consistent plasma parameters for a specified field configuration. The MHD momentum equation is given by

$$
\rho \frac{d \mathbf{v}}{d t}=-\nabla \cdot \mathbf{P}+\mathbf{J} \times \mathbf{B}+\rho_{c} \mathbf{E}+\rho \mathbf{g}
$$

where $\rho$ is the mass density, $\mathbf{v}$ is the bulk flow velocity, $\mathbf{P}$ is the plasma pressure tensor, $\mathbf{J}$ is the current density, $\mathbf{B}$ is the magnetic field, $\rho_{c}$ is the charge density, $\mathbf{E}$ is the electric field, and $\mathbf{g}$ is the gravitational acceleration. In our study, the electrical and gravitational forces are negligible compared with the other terms.

Since we are primarily interested in the field at quiet times, this allows further simplification of equation (1). In the static limit, the partial time derivative of the bulk flow velocity vanishes. As average bulk flow velocities are small, the convective derivatives of the velocities are typically an order of magnitude smaller than magnetic stresses. The remaining contributions are the magnetic stress and the pressure tensor divergence; their balance produces magnetohydrostatic equilibrium:

$$
\mathbf{J} \times \mathbf{B}=\nabla \cdot \mathbf{P}
$$

Bulk plasma properties, such as pressure, are calculated from a moment of the particle distribution function. We have chosen a two-temperature, bi-Maxwellian, equatorial distribution function. Liouville's theorem in conjunction with conservation of the first adiabatic invariant and conservation of energy allows us to describe how the distribution function maps along a flux tube from the equator. By taking the second moment of the mapped distribution function at a position, " $s$ ", along the flux tube, it can be shown that the pressures vary away from the equatorial position " 0 " according to

$$
\begin{gathered}
P_{\perp}(s)=P_{\perp}(0) g\left(\Theta, A_{o}\right) \\
P_{\|}(s)=P_{\|}(0) g\left(\Theta, A_{o}\right)^{\frac{1}{2}} \\
g\left(\Theta, A_{o}\right)=\left[\Theta+\left(\frac{1-\Theta}{A_{o}}\right)\right]^{-2}
\end{gathered}
$$

where $\Theta=B(0) / B(s)$ and $A_{o}=P_{\|}(0) / P_{\perp}(0)$ where $P_{\perp}\left(P_{\|}\right)$is the pressure perpendicular (parallel) to the magnetic field direction. For a bi-Maxwellian distribution of plasma, it can be shown from equations (3) and (4) that the pressure anisotropy maximizes at the equator and tends toward isotropy with increasing $s$.

With the assumption of a gyrotropic pressure [Chew et al., 1956], equation (2) may be written in the form

$$
\mathbf{J} \times \mathbf{B}=\nabla_{\perp} P_{\perp}+\left(P_{\|}-P_{\perp}\right) \frac{\mathbf{B}}{B} \cdot \nabla \frac{\mathbf{B}}{B}
$$

The parallel component of equation (2) gives no additional constraint; it is satisfied identically by equations (3) and (4). Both $\mathbf{B}$ and $\mathbf{J}\left[\mathbf{J}=\left(1 / \mu_{o}\right) \nabla \times \mathbf{B}\right]$ are determined directly from the TU models and are taken as givens. Therefore, the left-hand side and the magnetic field curvature on the right-hand side of equation (5) are input parameters of our calculations, whereas the components of the pressure are the unknowns to be determined.

\section{Determination of Pressure Gradients and Anisotropies}

Using equations (3), (4), and (5) we have determined the plasma pressures and anisotropies consistent with the TU magnetic field models. This section describes the "inversion" technique applied to the least active $\mathrm{TU}$ model $\left(K_{p}=0\right)$. The calculations were restricted to the midnight meridian in the near magnetotail region, a region well-described by the TU models. Flux tubes with equatorial crossing distances between $6.5 R_{E}$ and $12 R_{E}$ were considered. Calculations were done only for sections of the flux tubes at radial distances greater than $6.5 R_{E}$.

Solutions to equation (5) were found with a parameter search on the variables $P_{\perp}(0)$ and $\nabla_{\perp} P_{\perp}(0)$. Solutions were parameterized by the value of $P_{\perp}(0)$ on the innermost flux tube at $6.5 R_{E}$. The perpendicular pressure gradient at the equator was then chosen. Equation (5) shows these two equatorial variables determine $P_{\|}(0)$.

Although, by definition, the parameters satisfy equation (5) at the equator at the initial starting distance, there is no guarantee that equation (5) is satisfied off the equator. To obtain $\nabla_{\perp} P_{\perp}(s), P_{\perp}(s)$ and $P_{\mid}(s)$ were determined as a function of $s$ from equations (3)-(4) and the initial set of equatorial parameters $\left(\Theta, A_{o}\right.$, and $\left.P_{\perp}(0)\right)$. Field lines were traced by numerically integrating the field line equation using the TU model field. The same procedure was followed on nearby flux tubes separated from the initial flux tube by small spatial steps, $\Delta \mathbf{x}$, in the $\pm \mathbf{J} \times \mathbf{B}$ direction. To obtain equatorial values on nearby field lines we assumed a locally constant equatorial anisotropy and an expansion to first order in $\Delta \mathbf{x}$ of pressure $\left(P_{\perp}(0, x+\Delta x)=\Delta \mathbf{x} \cdot \nabla_{\perp} P_{\perp}(0, x)+\right.$ $\left.P_{\perp}(0, x)\right)$. Assuming that $A$ is locally constant is valid only for a nearly isotropic plasma; this assumption is justified by the solutions. Having mapped the perpendicular pressure along several closely-spaced flux tubes, a cubic spline curve was fit through the perpendicular pressures at $0.1 R_{E}$ intervals along the flux tube. In this manner, we determined the local perpendicular pressure gradient, $\Gamma_{\perp} P_{\perp}(s)$, by calculating the first-order variation of $P_{\perp}$ in the perpendicular direction. The procedure was repeated for a large range of assumed values of $\nabla_{\perp} P_{\perp}(0)$ for fixed $P_{\perp}(0)$ on the innermost flux tube.

The value of $\nabla_{\perp} P_{\perp}(s)$ from the spline fit was then compared with the value obtained directly from equation (5) (denoted $\mathrm{G}(s)$ ), at $0.1 R_{E}$ intervals along $s$. The fractional error, $e(s)=\left|\nabla_{\perp} P_{\perp}(s)-\mathbf{G}(s)\right| /\left|\nabla_{\perp} P_{\perp}(s)\right|$ was obtained at each point. The optimal $\nabla_{\perp} P_{\perp}(0)$ was taken as the one that gave a minimum of the fractional 


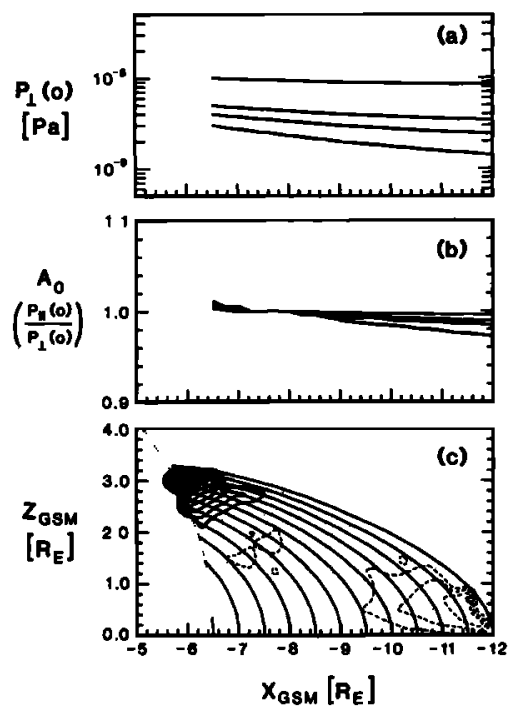

Fig. 1. Panel (a) shows four solutions of the calculated $P_{\perp}(0)$ as a function of distance in the magnetic equatorial plane in the midnight meridian. Panel (b) shows the corresponding equatorial pressure anisotropies $\left(P_{\|} / P_{2}\right)$. Panel (c) shows contours of constant percent fractional error, $e(s)$ (see text for definition), for a typical solution. The contour increment is $\pm 5 \%$.

error averaged over all points along the flux tube. Having chosen $\nabla P_{\perp}(0)$ for the innermost flux tube, a linear extrapolation of the solution was used to estimate $P_{\perp}(0)$ on the next downtail flux tube, and the entire procedure was repeated. The calculation was continued until the flux tube at $12 R_{E}$ was reached.

The solution is parameterized by $P_{\perp}(0)$ at the innermost flux tube. Therefore, the calculations were repeated for different initial $P_{\perp}(0)$ 's to obtain a family of solutions. We found that not all solutions are physically reasonable. For example equation (5) could be satisfied with a pressure gradient so large that the pressure became unacceptably small within $12 R_{E}$. Some solutions gave anisotropies that were unstable to the MHD mirror or fire-hose instabilities, unacceptable in an equilibrium configuration. Finally, the average fractional error varied significantly for different choices of $P_{\perp}(0)$. Thus, the number of possible solutions was narrowed to the physically acceptable ones with small average fractional errors. Solutions for $K_{p}=0$ are shown in Figure 1. Figure 1a shows the variation of $P_{\perp}(0)$ as a function of equatorial distance in the midnight meridian. Several solutions are indicated for different values of $P_{\perp}(0)$ at $6.5 R_{E}$. The top and bottom curves set approximate upper and lower bounds on allowable solutions. Figure 1b illustrates the corresponding variation of $A_{0}$. One consequence of a nearly isotropic pressure is that the magnetic tension must be balanced predominantly by the perpendicular pressure gradient. Thus, it is evident why a family of solutions is found. Figure 1a illustrates that for each allowable solution, nearly equivalent pressure gradients are found.

To illustrate the consistency of the model we show a cut through the midnight meridian $\left(X_{G S M}\right.$ vs. $Z_{G S M}$ ), in Figure 1c. Smooth unbroken curves represent the field lines threading this region and a dotted curve marks the innermost calculation boundary at $6.5 R_{E}$. The highest latitude field line and the dotted curve bound the calculation region. Superimposed are contours of constant fractional error (previously defined but here as a percentage) of a typical solution. Figure 1c indicates that typical solutions exhibit very small fractional error $(<5 \%)$ throughout nearly all of the calculation region, having significant error $(>20 \%$ ) only in a small region away from the equator. Furthermore, the error is nowhere larger than $50 \%$. The consistency between the field model and the currents produced by our simple model plasma suggest that during quiet conditions, the low geomagnetic activity $\mathrm{TU}$ models very nearly approach the condition of magnetohydrostatic equilibrium in the near-tail region. We also find that the errors in fits of bi-Maxwellian plasma distributions to the higher $K_{p}$ models are always greater than the $K_{p}=0$ model, although the qualitative results remain the same. We have not identified quantitatively the sources of the larger errors but several are obvious. Our model neglects bulk flow velocities. Furthermore, time variations, present during more active conditions, are averaged out in the $\mathrm{TU}$ models and have not been included in our analysis.

\section{Comparison with Data}

The quiet time solutions for $P_{\perp}(0)$ and $A_{0}$ can be used to test the self-consistency of the TU model with the requirements of magnetohydrostatic equilibrium. For this purpose, we compare the dominant features of the solutions (near isotropy and pressure gradients, $\left|\nabla_{\perp} P_{\perp} / P_{\perp}\right|$, of order $0.1-0.2 / R_{E}$ ) with in situ observations of these parameters under like conditions. Stiles et al. [1978] investigated the quiet time plasma sheet anisotropies of protons using the IMP 6 and IMP 8 spacecraft. In the region of our calculations, they found that the anisotropies differed from unity by less than the probable error in the measurement. Thus, they established that in the nearearth plasma sheet, the particle population carrying the bulk of the energy density is essentially isotropic. Figure $1 \mathrm{~b}$ demonstrates that the TU model is consistent with a pressure anisotropy differing from unity by less than $2 \%$.

We would also like to compare the calculated and observed pressure gradients. Unfortunately, there have been no systematic statistical surveys of the plasma pressure in the regions of interest. There have been few spacecraft that measure enough of the proton distribution in the near-tail vicinity to yield the plasma pressure accurately. Recently, the AMPTE/CCE spacecraft has filled in the gap for the region of the ring current and inner magnetosphere. We can compare our results with CCE data to $8.8 R_{E}$, the spacecraft apogee.

Unfortunately, the published CCE data available for comparison were obtained during relatively active periods. Therefore, we have compared the observations with the results of our calculations for the most disturbed $\mathrm{TU}$ model $\left(K_{p}>3^{+}\right)$. As noted before, the errors are larger for the most disturbed TU models, but even so the local value of percent $e(s)$ is everywhere smaller than $60 \%$ and the average percent error is always less than $30 \%$. Figure 2 compares the pressure obtained for the disturbed TU 


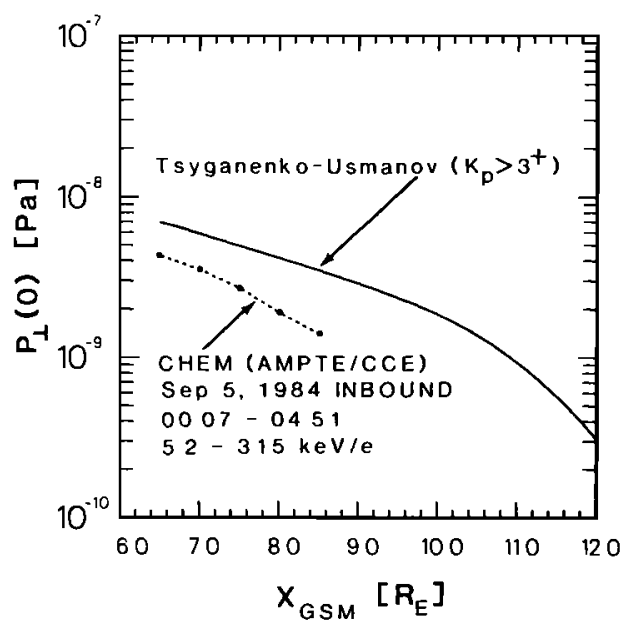

Fig.2. Pressure versus downtail distance for the active TU model (solid curve) in the midnight meridian and in situ measurements from the CHEM instrument on the CCE spacecraft (dashed curve) during disturbed time (taken from Williams [1987]). Despite differences inherent to both curves (see text), the correspondence between the pressure gradients is reasonably good.

model with the pressure distribution determined by the CHEM instrument on AMPTE/CCE. Note that our pressure represents an averaged distribution while the CHEM pressure, derived from ions with energies between 5.2 and $315 k \epsilon V / e$, is from a single pass through the region. Furthermore, our calculations are for the midnight meridian whereas the CHEM data were gathered on an orbit that started nearer to dawn and ended near midnight.

Although the pressure magnitudes differ considerably, the gradients are similar especially inside of $7.5 R_{E}$. We have noted above that the pressure gradient and not the absolute magnitude of the pressure is important for a nearly isotropic plasma. Our calculations determine the total self-consistent plasma pressure whereas the measured pressure is only a lower bound. It may be significant that the magnitude of the measured pressure falls off with distance faster than does the model pressure. As the spacecraft moves to greater radial distance, the peak of the proton distribution shifts to lower energy and a larger portion of the distribution lies below the lowest energy measured by CHEM. Thus, at greater distances the pressure measured by CHEM represents a smaller fraction of the total plasma pressure. In view of the limitations of the data and the doubtful accuracy of a statistical model for a disturbed magnetosphere, we find the correspondences between model and observation to be quite good. We look forward to obtaining complete quiet time data that will provide better tests.

\section{Summary}

We have developed a technique of "inverting" an empirical magnetic field model to determine the self-consistent plasma properties. The technique has been applied to a set of recently developed terrestrial magnetic field models. We regard the agreement between model plasma parameters and the limited data available as satisfactory. Given the initial success of the method, we suggest that the predictions of our calculations be further tested and that the technique be applied to other magnetic field models, either observed or theoretical, to aid in understanding the interactions of field and plasma in an equilibrium magnetosphere.

Acknowledgements. We wish to thank David P. Stern for supplying us with the coded version of the TU magnetic field models and G.-Hannes Voigt for helpful discussions. This work was partially supported by the National Science Foundation, division of Atmospheric Sciences, under grants ATM 83-00523 and ATM 86-10858. One of the authors (HES) is supported by an Office of Naval Research graduate fellowship.

\section{References}

Chew, G. F., M. L. Goldberger, and F. E. Low, The Boltzmann equation and the one-fluid hydromagnetic equations in the absence of particle collisions, Proc.Roy.Soc., A236, 112, 1956.

Stiles, G. S., E. W. Hones, S. J. Bame, and J. R. Asbridge, Plasma sheet pressure anisotropies, J. Geophys. Res., 83, 3166, 1978.

Tsyganenko, N. A., and A. V. Usmanov, Determination of the magnetospheric current system parameters and development of experimental geomagnetic field models based on data from IMP and HEOS satellites, Planet. Space Sci., 30, 985, 1982.

Voigt, G. H., A mathematical magnetospheric field model with independent physical parameters, Planet. Space. Sci., 29, 1, 1981.

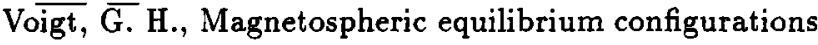
and slow adiabatic convection, in Solar Wind-Magnetosphere Coupling, edited by Y. Kamide and J. A. Slavin, pp. 233-273, Terra Scientific Publishing Company, Tokyo, 1986.

Walker, R. J., and D. J. Southwood, Momentum balance and flux conservation in model magnetospheric magnetic fields, J. Geophys. Res., 87, 7460, 1982.

Williams, D.J., The earth's ring current: present situation and future thrusts, Physica Scripta, 35, (in press), 1987.

(Received April 2, 1987 ; revised June 10, 1987 ; accepted June 22, 1987.) 